\title{
Bacterial morphologies supporting cometary panspermia: a reappraisal
}

\author{
Chandra Wickramasinghe \\ Cardiff Centre for Astrobiology, Cardiff University, Cardiff, UK \\ e-mail:ncwick@googlemail.com
}

\begin{abstract}
It is nearly 30 years since the first decisive evidence of microbial morphologies in carbonaceous chondrites was discovered and reported by Hans Dieter Pflug. In addition to morphology, other data, notably laser mass spectroscopy, served to confirm the identification of such structures as putative bacterial fossils. Recent examinations of cometary dust collected in the stratosphere and further studies of carbonaceous meteorites reaffirm the presence of putative microbial fossils. Since carbonaceous chondrites (particularly Type 1 chondrites) are thought to be extinct comets the data reviewed in this article provide strong support for theories of cometary panspermia.

Received 24 April 2010, accepted 30 April 2010

Key words: panspermia, comets, meteorites, microfossils.

'Microbiology may be said to have had its beginnings in the nineteen-forties. A new world of the most astonishing complexity began then to be revealed. In retrospect I find it remarkable that microbiologists did not at once recognise that the world into which they had penetrated had of necessity to be of a cosmic order. I suspect that the cosmic quality of microbiology will seem as obvious to future generations as the Sun being the centre of our solar system seems obvious to the present generation'
\end{abstract}

- Fred Hoyle The Relation of Biology to Astronomy (University College Cardiff Press, 1980)

\section{Introduction}

The amazingly rich diversity of extraterrestrial organics recently discovered in the Murchison meteorite (SchmittKopplin et al. 2010) comes as no surprise in the context of cometary panspermia. If cometary bodies are the carriers of microbial life, a diversity of organic molecules as rich as or possibly richer than that which prevails naturally on the Earth is to be expected. Moreover, such molecules may have been generated overwhelmingly through biochemical rather than abiotic or prebiotic processes (N.C. Wickramasinghe 2010). Since an extraterrestrial biology carried in comets would be expected to span a far greater range of possibilities than the limited subset selected by niches available on the Earth, a higher level of diversity in the extraterrestrial organics is not at all surprising. It may not be necessary to assign such a diversity to prebiotic processes as suggested by Schmitt-Koplin. For instance, 'non-biological' amino acids e.g. AIB and isovaline - that might be relevant to an exotic or alien biology were found at the $\mathrm{K} / \mathrm{T}$ boundary, and it has been argued that this material may have been deposited by comets (Wallis 2007). Similarly, the range of molecules discovered in the Murchison meteorite could include degradation products of a wide range of alternative non-terrestrial biologies.
Primordial cometary bodies endowed with radioactive heat sources provide an ideal venue not only for the replication of microbial life once it has started, but also for an ab initio origin of life at some earlier stage (Napier et al. 2007; J.T. Wickramasinghe et al. 2010; Gibson \& Wickramasinghe 2010; Gibson et al. 2010). Within an individual cometary body endowed with nutrients and chemical energy, a preexisting microbiota can proliferate on a very short timescale (Hoyle \& Wickramasinghe 1981, 1982, 1985). Thereafter, an amplified microbial population becomes locked in a freezedried state until the comet comes to be peeled away layer by layer, thus releasing viable microbes into space (Hoyle \& Wickramasinghe 1985). The expulsion of volatiles from a comet over many perihelion passages would eventually lead to a compaction of mineral grains in comets along with residual microorganisms that effectively 'sediment' and fossilize. On this basis, it is possible to understand an origin of Type I carbonaceous chondrites such as the Murchison and Orgueil meteorite as fragments of 'extinct' comets.

\section{Organized elements in meteorites}

An intriguing controversy followed reports by Claus and Nagy (1961) and Urey (1966) in which it was claimed that microbial fossils are present in several carbonaceous 

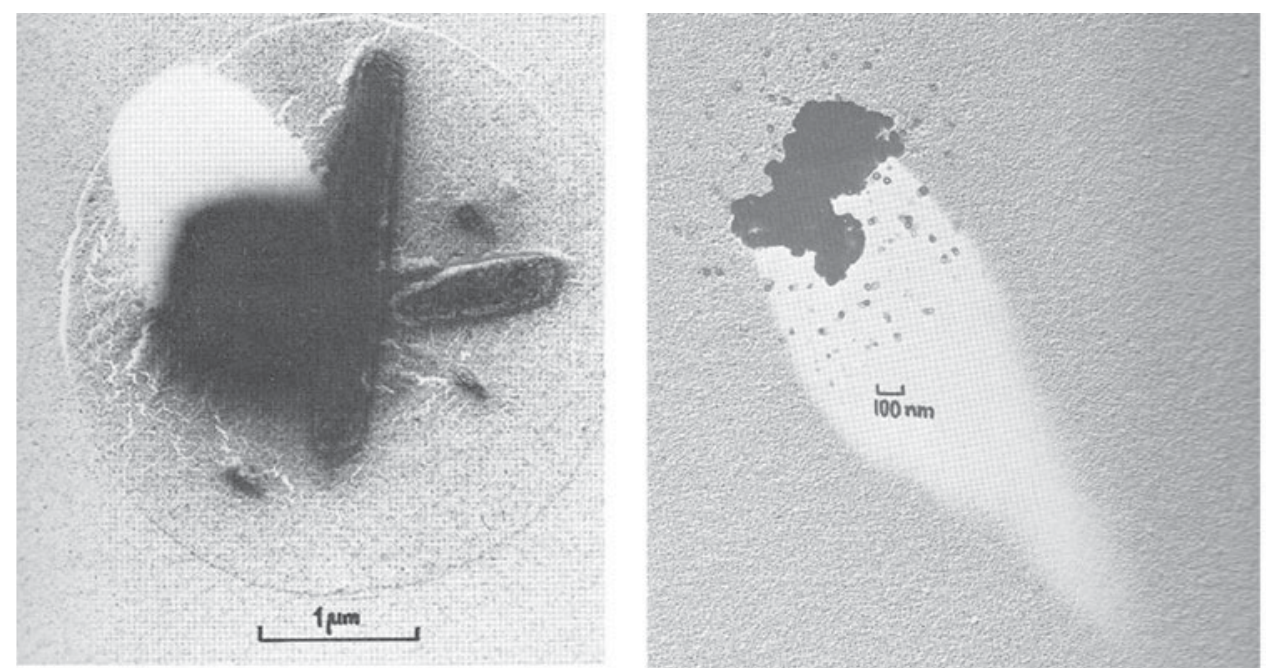

Fig. 1. Particles in the atmosphere recovered by E.K. Bigg from heights above $25 \mathrm{~km}$ (from Bigg 1984). The left-hand frame shows particles resembling bacilli in a clump within a droplet of sulphuric acid. The right-hand frame shows a carbonaceous particle releasing a cloud of viral sized particles on exposure to an electron beam.

chondrites. Although electron micrographs revealed evidence of structures resembling cell walls and flagellae and a wide range of biological morphologies, all these identifications came to be discredited because contamination by ragweed pollen was demonstrated in at least one instance. It was clear that much more careful attention to detail was needed before this matter could advance any further.

Independent work carried out in the 1960 s by E.K. Bigg involved collection of atmospheric particles above an altitude of $25 \mathrm{~km}$ which also revealed structures that were similar to microorganisms when examined under a microscope (Bigg 1984). Although terrestrial contamination (lofting from the surface) could not be ruled out at this altitude, such structures as shown in Fig. 1 could be consistent with the infall of micrometeorites with entrapped extraterrestrial microbes. The image in the right-hand panel of Fig. 1 shows viral-sized particles being expelled when an original carbonaceous particle is exposed to an electron beam. This early pioneering work also called for a repetition with more stringent controls and constraints so as to minimize the possibility of contamination. Confirmation of elemental and molecular composition consistent with biology was also needed, and techniques to achieve this were not readily available at the time.

Nearly two decades later the problem of microbial fossils in carbonaceous meteorites was re-examined by Hans D. Pflug with special attention being paid to avoid the criticisms of earlier work (Pflug 1984). Pflug used state-of-the-art equipment to prepare ultra-thin sections $(<1 \mathrm{~mm})$ of the Murchison meteorite in a contaminant free environment.

Thin slices of the Murchison meteorite were placed on membrane filters and exposed to hydrofluoric acid vapour. In this way in situ demineralization was achieved, the mineral component being removed though the pores of the filter, leaving carbonaceous structures indigenous to the meteorite intact. A wealth of morphologies with distinctive biological characteristics was thus revealed. Two examples are shown in Figs 2 and 3. Figure 2 shows structures uncannily similar to a well-known bacterium pedomicrobium, and Fig. 3 displays a clump of nanometre-sized particles with internal structure similar to a modern influenza virus. In view of the techniques used in the preparation of the slides, it could be asserted with confidence that all these structures are indigenous to the meteorite, not contaminants.

Microprobe analysis using laser mass spectroscopy, Raman spectroscopy, ultraviolet and infrared spot spectroscopy were used to determine composition as well as to establish the indigenous nature of individual particles. Pflug's laser mass spectrum analysis on one of these particles is shown in Fig. 4. From Fig. 4, with many of the peaks yet to be unambiguously identified, we see that the particles with these biological-type morphologies also have chemical signatures fully consistent with degraded or fossilized microbial matter. Indeed comparisons of these structures with well-recognized microbial fossils in the Gunflint cherts showed nearly identical results in laser mass spectroscopy, demonstrating that the same organic functional groups were present in both situations. Further work by Pflug \& Heinz (1997) confirmed these results and the criticism of contamination levelled against Claus and Nagy now became largely irrelevant. A more detailed re-appraisal of mass peaks in the data of Pflug and his collaborators is currently in progress (Wallis et al. 2010).

Pflug's pioneering work was confirmed and amplified in large measure by Richard Hoover and his collaborators from 2000 to the present day (Rozanov \& Hoover 2003; Hoover 2005). Hoover and colleagues showed that diverse microbial morphologies were present on freshly fractured surfaces of carbonaceous chondrites, including Murchison and Tagish Lake meteorites. They consider various criteria for separating recent terrestrial contamination from indigenous microfossils and have diligently compiled an atlas of prospective bacterial fossils. Backscatter electron images at high resolution in 


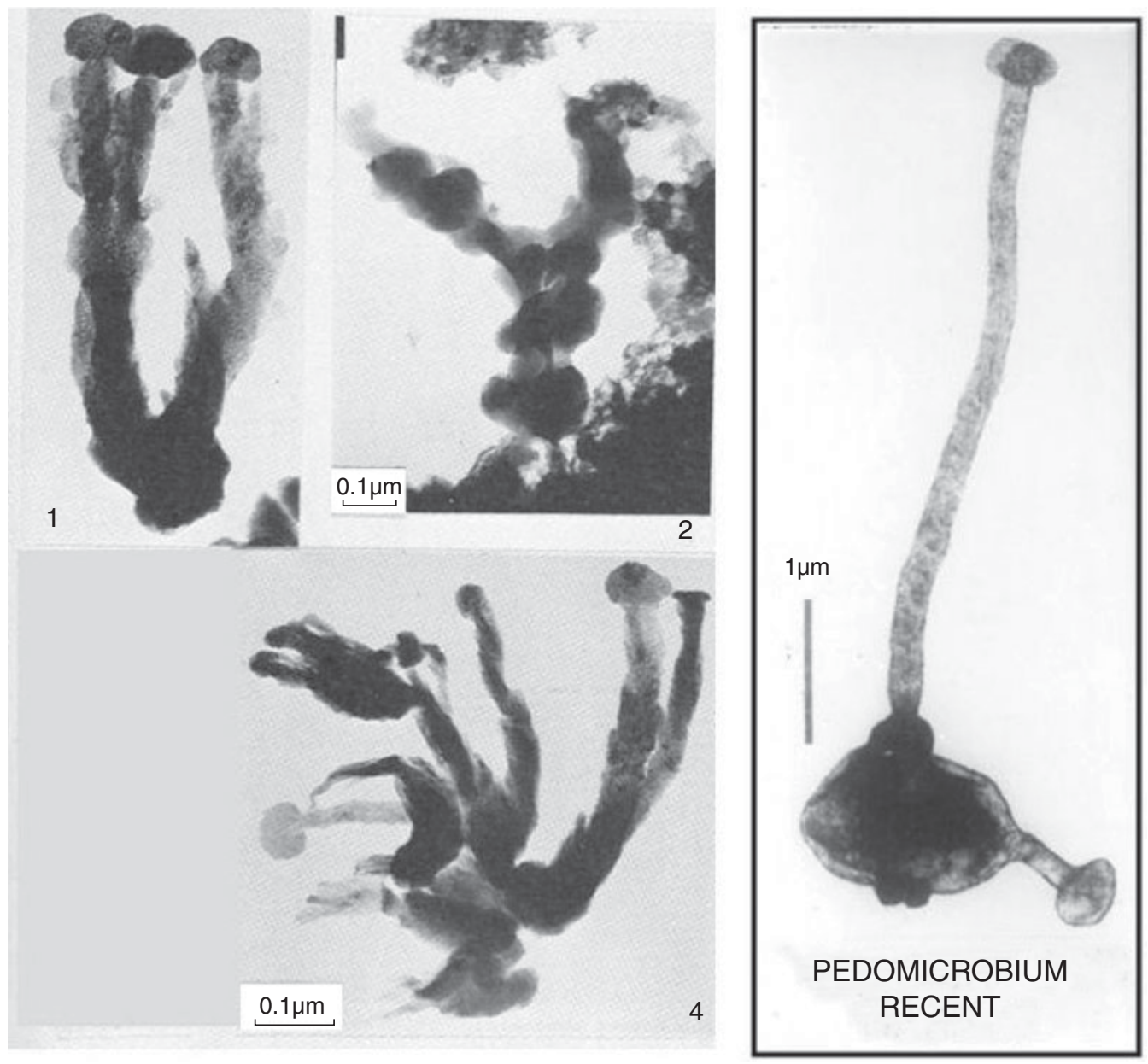

Fig. 2. The comparison of a characteristically biological structure in the Murchison meteorite with a similar structure corresponding to a modern iron-oxidizing microorganism - pedomicrobium.

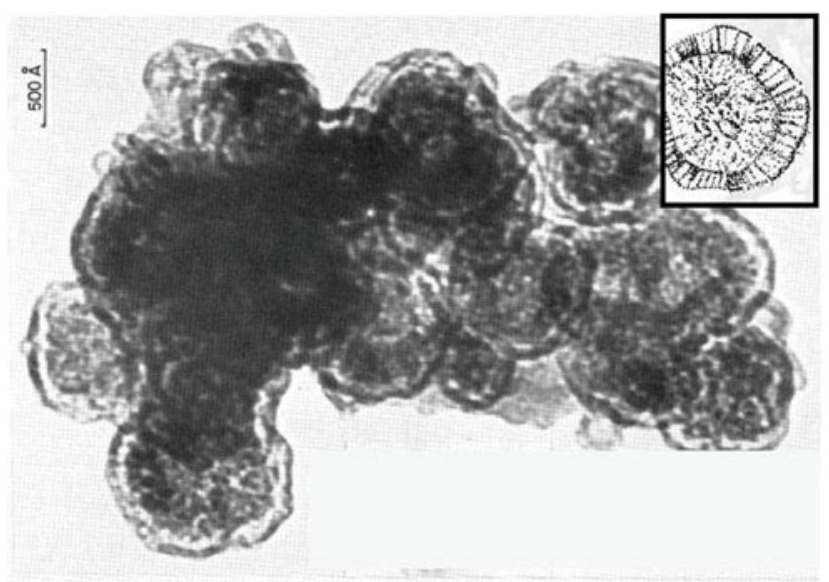

Fig. 3. An electron micrograph of a structure resembling a clump of viruses - influenza virus - also found in the Murchison meteorite. The drawing in the inset is a representation of a modern influenza virus displaying astounding similarities in structure to the putative clump of fossil viruses.

Fig. 5 show a particularly impressive example comparing indigenous structures on a freshly cleaved surface of the Murchison meteorite with living cyanobacteria.

\section{Microfossils in cometary dust}

On a much smaller scale than meteorites, clumps of interplanetary dust particles of cometary origin have been collected by D.E. Brownlee and his colleagues over many years (Brownlee 1978). The technique was to use sticky paper flown on U2 aircraft sweeping up large quantities of aerosols from which interplanetary particles have to be diligently separated from terrestrial contaminants. These so-called Brownlee particles, which were mostly in the form of fluffy aggregates of siliceous dust, have been found to contain extraterrestrial organic molecules, with a complexity and diversity approaching that recently reported for the Murchison meteorite (Clemett et al. 1993). In a few instances, microbial morphologies were discovered within individual Brownlee particles.

Figure 6 shows such a micrometre-sized carbonaceous structure in a Brownlee particle compared with a microbial fossil-an iron oxidizing microorganism-found in the Gunflint cherts of N. Minnesota. The striking similarity seen here once again argues in favour of a biological rather than an abiotic explanation for the extraterrestrial particle (Bradley et al. 1984; Hoyle et al. 1985).

Cometary dust from comet Wild 2 collected in highvelocity impacts with aerogel in the Stardust Mission 


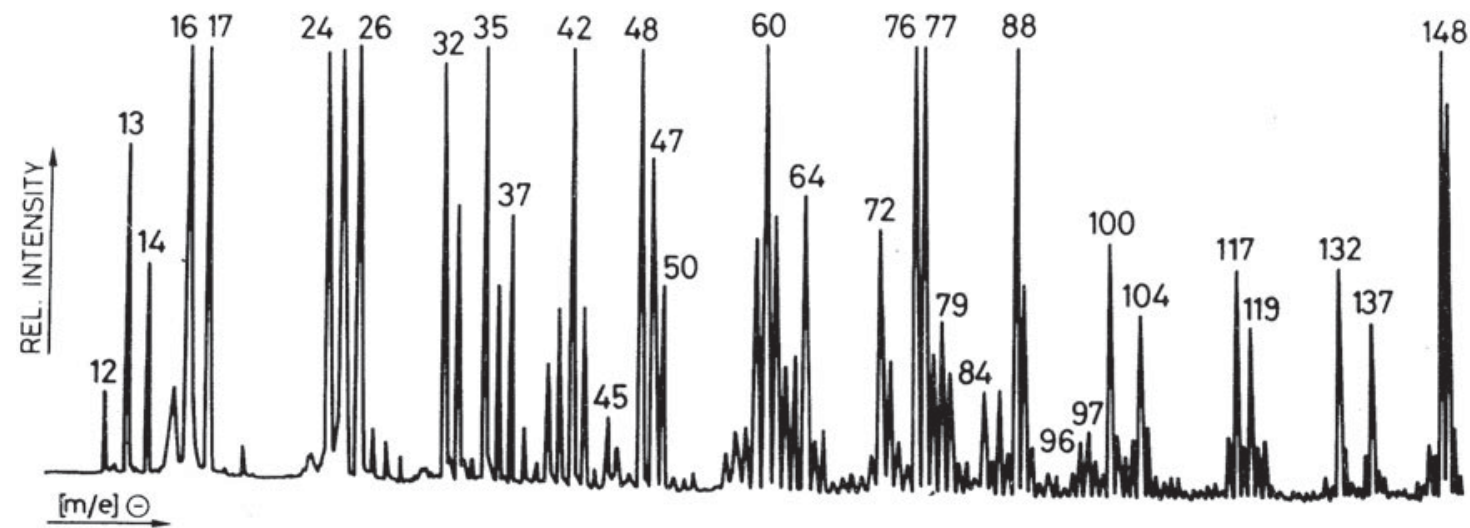

Laser mass spectrum (negative ions) from individual particle in thin section of the Murchison meteorite. Field of measurement ca. $1 \mu \mathrm{m}$. Attribution of signals: 12: $\mathrm{C}^{-}$, 13: $\mathrm{CH}^{-}, 14: \mathrm{CH}_{2}^{-}, 16: \mathrm{O}^{-}, 17: \mathrm{OH}^{-}, 19: \mathrm{F}^{-}, 24: \mathrm{C}_{2}^{-}, 25: \mathrm{C}_{2} \mathrm{H}^{-}, 26: \mathrm{CN}^{-}, 28: \mathrm{Si}^{-}, 32: \mathrm{S}^{-}$, $35: \mathrm{Cl}^{-}, 36: \mathrm{C}_{3}^{-}, 37: \mathrm{C}_{3} \mathrm{H}^{-}, 40-42,45:$ fragmental carbonaceous groups, $48: \mathrm{C}_{4}^{-}, 49: \mathrm{C}_{4} \mathrm{H}^{-}$, $50: \mathrm{C}_{4} \mathrm{H}_{2}^{-}, 60: \mathrm{SiO}_{2}^{-}$resp. $\mathrm{C}_{5}^{-}, 61: \mathrm{C}_{5} \mathrm{H}^{-}, 72: \mathrm{C}_{6}^{-}, 73: \mathrm{C}_{6} \mathrm{H}^{-}, 76: \mathrm{SiO}_{3}^{-}, 96: \mathrm{C}_{8}^{-}, 97: \mathrm{C}_{8} \mathrm{H}^{-}$, 108: $\mathrm{C}_{9}^{-}, 120: \mathrm{C}_{10}^{-}, 121: \mathrm{C}_{10} \mathrm{H}^{-}$. (Analysis and interpretations: H. J. Heinen, K.-D. Kupka).

Fig. 4. Laser mass spectrum reproduced from Pflug (1984).

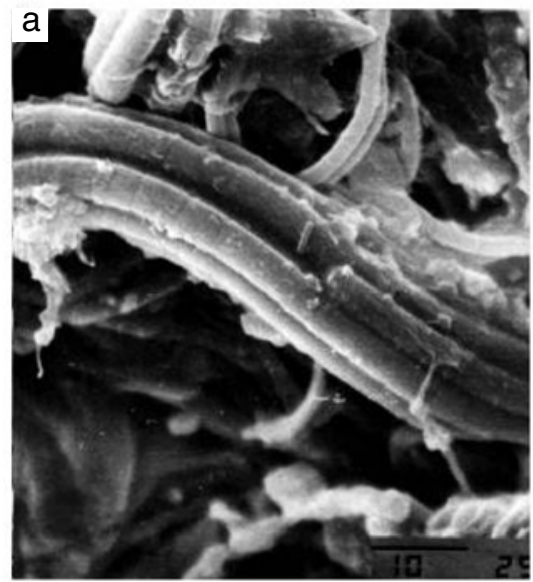

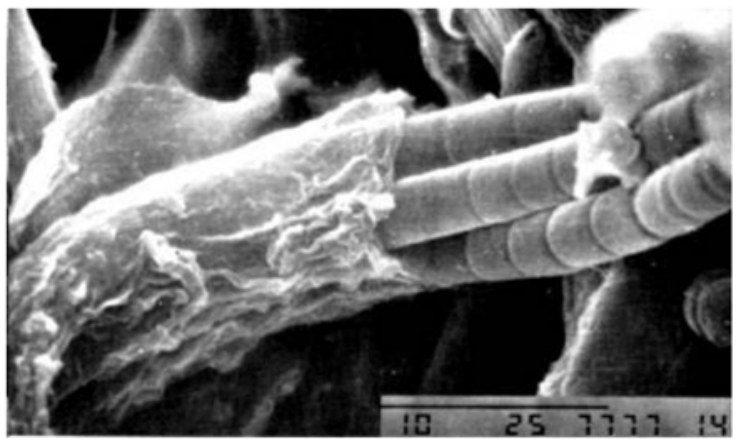

Living cyanobacteria
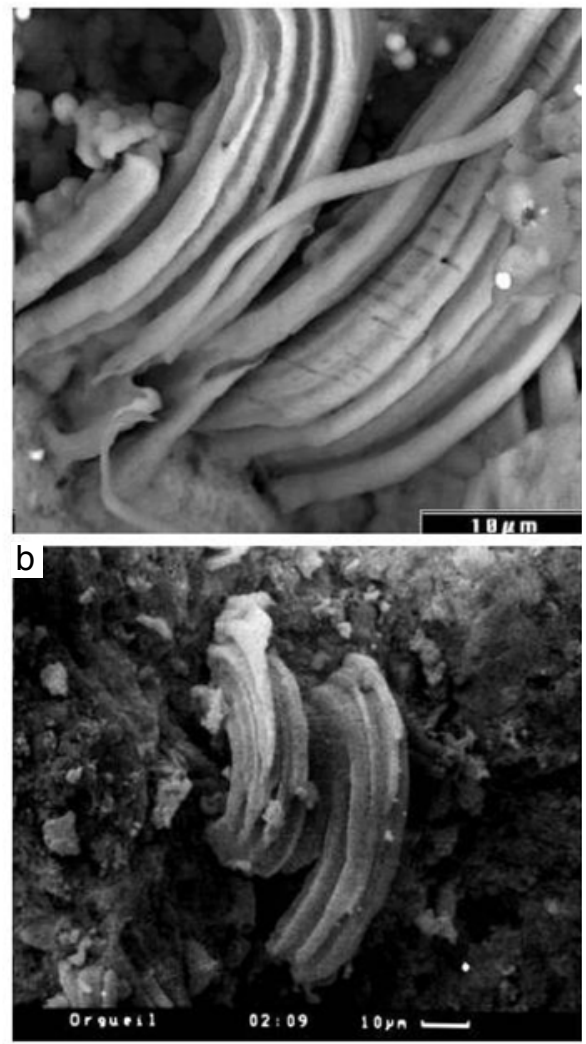

Microfossils in Murchison

Fig. 5. Structures in the Murchison meteorite (Hoover 2005) compared with living cyanobacteria.

would be expected to have a somewhat lower level of molecular diversity and complexity than the samples studied by Clemett et al. (1993), and fragile biological structures would not have been recovered intact. This is indeed borne out in examinations of Stardust material (Sandford et al. 2006). 
Since 2001 pristine cometary dust was collected aseptically using cryoprobes flown aboard balloons to heights of $41 \mathrm{~km}$ (Harris et al. 2002; Narlikar et al. 2003; Wainwright et al. 2004). Amongst the aerosols collected was a rich harvest of pristine carbonaceous cometary dust with morphologies generally similar to bacterial fossils.

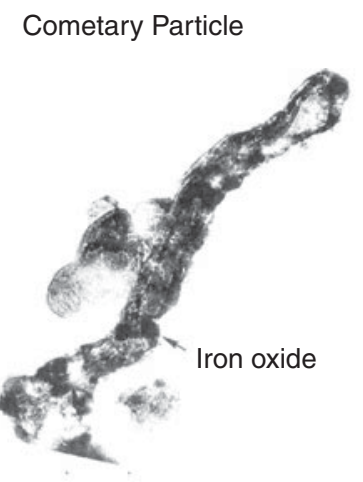

Terrestrial Bacterial Fossil

\section{$\overline{0.1 \mu \mathrm{m}}$}

Fig. 6. An organic particle in a Brownlee clump compared with a terrestrial bacterial fossil (Hoyle et al. 1985).
A sample of putative bacterial fossils from samples collected in the stratosphere in 2001 is shown in the scanning electron microscope images of Fig. 7. Morphological similarities to cocoidal and rod-shaped bacteria have been noted by several investigators (Harris et al. 2002; Wainwright et al. 2008; Rauf et al. 2010). In a few instances, evidence of fimbrae and biofilm appear to corroborate a biological interpretation, and in all cases shown here energy dispersive $\mathrm{X}$-ray analyses have shown high $\mathrm{C}$ abundances (Wainwright et al. 2008). The height of $41 \mathrm{~km}$ from which the collections were made is arguably too high for lofting a $10 \mu \mathrm{m}$ sized clump of bacteria from the surface, so structures such as those seen in Fig. 7 can be argued to represent infalling cometary dust.

In addition to either dormant or 'fossil' microorganisms represented in Fig. 6, evidence of viable but non-culturable clumps of living bacteria were found as seen in the staining image of Fig. 8 (Harris et al. 2002). A few cultures of microorganisms were also reported (Wainwright et al. 2003; Narlikar 2010), but their origin still remains uncertain. The criticism that these microorganisms cannot be derived from comets because of their genetic similarity to terrestrial microbes is highly questionable (Vaidya 2009). If evolution of life on Earth involves the continual assimilation of new genes
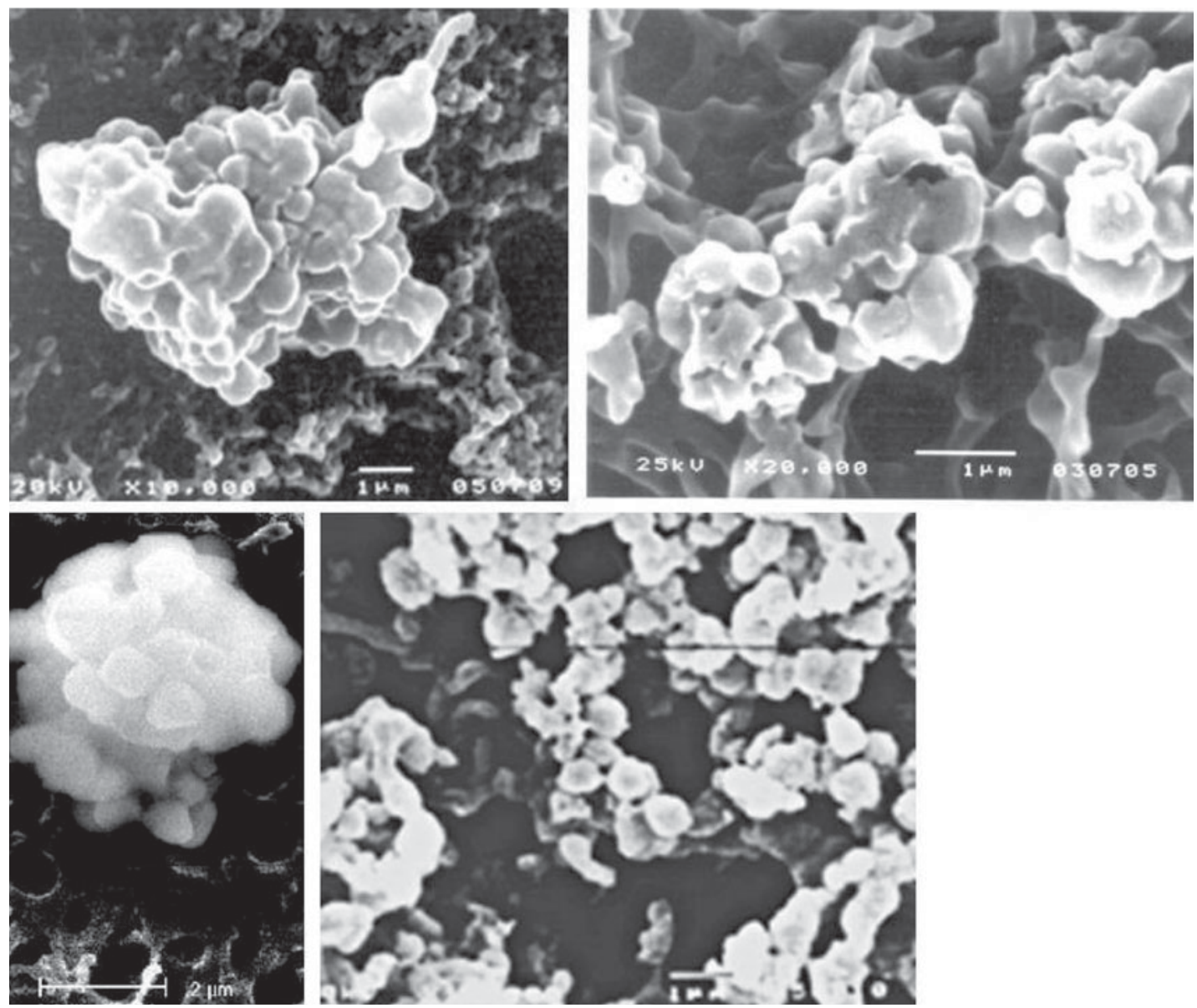

Fig. 7. Putative microbial fossils in stratospheric aerosols (Harris et al. 2002). 


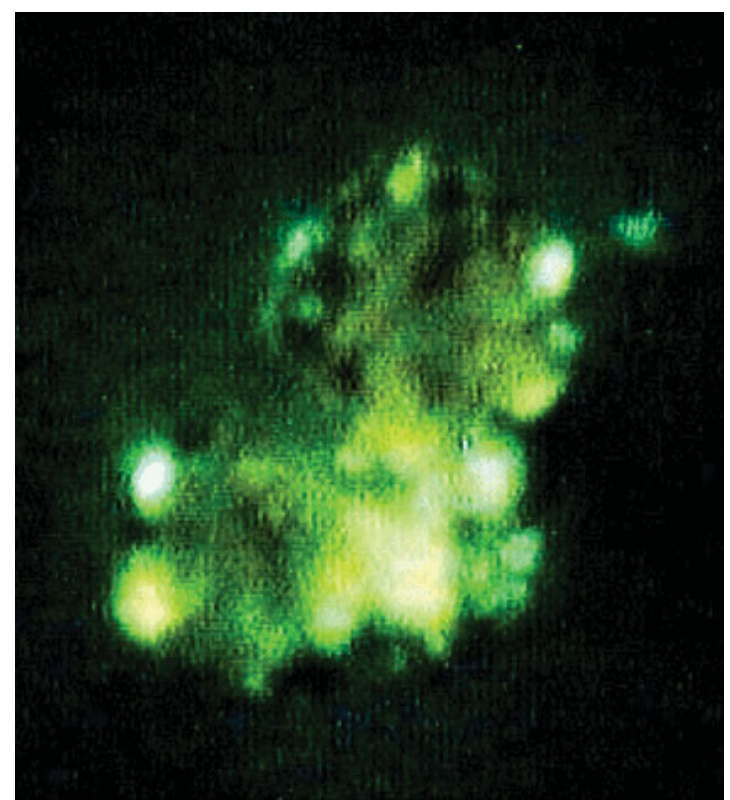

Fig. 8. A clump of living bacteria recovered in the 2001 stratospheric collection fluorescing with carbocyanine dye.

from cometary microbes (Hoyle \& Wickramasinghe 1982) the argument of Vaidya fails. Genetic proximity between newly arriving cometary microbes and microorganisms already established on the Earth would indeed be expected on this basis.

\section{Concluding remarks}

In view of the recent revival of interest in the theory of cometary panspermia (Wickramasinghe 2010) a careful re-assessment of all its predictions now appears timely. Cometary panspermia argues that comets are the carriers and distributors of cosmic life as well as the sites of replication of cosmic bacteria. Since carbonaceous chondrites may be regarded as being the end product of the evolution of comets, progressively denuded of volatiles after very many perihelion passages, such objects would naturally be expected to carry chemical and morphological signatures of life.

Early reports in the 1960s purporting to find microfossils (organized elements) in meteorites came to be quickly discounted after a few instances of contamination by ragweed pollen contamination were discovered. This was an unfortunate development because later attempts to re-open the subject of microfossils in meteorites became inevitably tainted with prejudice. In this article a sample of the most convincing results obtained by Hans Pflug, Richard Hoover and others are assembled. From Figs $2-6$ it could be seen that microfossils in meteorites could provide strong-even compelling - support for the theory of cometary panspermia.

\section{References}

Bigg, E.K. (1984). Particles in the upper atmosphere. In Fundamental Studies and the Future of Science, ed. Wickramasinghe, N.C., pp. 38-51. University College Cardiff Press, Cardiff.

Bradley, J.P., Brownlee, D.E. \& Fraundorf, R. (1984). Science 223, 56-58.

Brownlee, D.E. (1978). Microparticle studies by sampling techniques. In Cosmic Dust, ed. McDonnell, J.A.M., pp. 295-336. Wiley Interscience, New York.

Claus, G. \& Nagy, B. (1961). Nature 192, 594-596.

Clemett, S.J. et al. (1993). Science 262, 721-725.

Gibson, C.H. \& Wickramasinghe, N.C. (2010). J. Cosmology 5, 1101-1120.

Gibson, C.H., Schild, R. \& Wickramasinghe, N.C. (2010). In preparation, arXiv:1004.0504.

Harris, M.J. et al. (2002). Proc. SPIE 4495, 192-198.

Hoover, R.B. (2005). Perspectives in Astrobiology, ed. Hoover, R.B., Rozanov, A.Y. \& Paepe, R.R., 366, 43. IOS Press, Amsterdam.

Hoyle, F. \& Wickramasinghe, N.C. (1981). Comets and the Origin of Life, ed. Ponnamperuma, C., p. 227. D. Reidel, Dordrecht.

Hoyle, F. \& Wickramasinghe, N.C. (1982). Proofs that Life is Cosmic. Govt. Press, Sri Lanka, Colombo (http://www.astrobiology.cf.ac.uk/ proofs...pdf)

Hoyle, F. \& Wickramasinghe, N.C. (1985). Living Comets. University College Cardiff Press, Cardiff.

Hoyle, F., Wickramsinghe, N.C. \& Pflug, H.D. (1985). Astrophys. Space Sci. 113, 209-210.

Napier, W.M., Wickramasinghe, J.T. \& Wickramasinghe, N.C. (2007). Int. J. Astrobiol. 6, 321-323.

Narlikar, J.V. (2010). Scientific American India April, 37-40.

Narlikar, J.V., Wickramasinghe, N.C., Wainwright, M. \& Rajaratnam, P. (2003). Current Science 85, 29.

Pflug, H.D. (1984). Utrafine structure of the organic matter in meteorites. In Fundamental Studies and the Future of Science, ed. Wickramasinghe, N.C., pp. 24-37. University College Cardiff Press, Cardiff.

Pflug, H.D. \& Heinz, B. (1997). Proc SPIE 3111, 86-97.

Rauf, K., Hann, A., Wallis, M. \& Wickramasinghe, C. (2010). Int. J. Astrobiol. 9(3), 183-189.

Rozanov, A.Yu \& Hoover, R.B. (2003). Proc. SPIE 5163, 23-35.

Sandford, S.A. et al. (2006). Science 314, 1720.

Schmitt-Kopplin, P. et al. (2010). Proc. Nat. Acad. Sci. 107, 2763-2768.

Urey, H.C. (1966). Science 151, 157-165.

Vaidya, P.G. (2009). Aperion 16, 463-474.

Wainwright, M., Wickramasinghe, N.C., Narlikar, J.V. \& Rajaratnam, P. (2003). FEMS Microbiol. Lett. 218, 161-165.

Wainwright, M., Wickramasinghe, N.C., Narlikar, J.V. \& Rajaratnam, P. (2008). Microbiol. Comment, DOI 10.1099/mic.0.26907.0.

Wainwright, M., Wickramasinghe, N.C., Narlikar, J.V., Rajaratnam, P. \& Perkins, J. (2004). Int. J. Astrobiol. 3, 13-15.

Wallis, M.K. (2007). Int. J. Astrobiol. 6, 303-306.

Wallis, J., Heinz, B. \& Wickramasinghe, N.C. (2010). In preparation.

Wickramasinghe, J.T., Wickramasinghe, N.C. \& Napier, W.M. (2010). Comets and the Origin of Life. World Scientific Publishing, Singapore.

Wickramasinghe, N.C. (2010). Int. J. Astrobiol. 9(2), 119-129. doi:10.1017/ S1473550409990413. 